\title{
LINC00152 upregulates ZEB1 expression and enhances epithelial-mesenchymal transition and oxaliplatin resistance in esophageal cancer by interacting with EZH2
}

Shuyao Zhang ${ }^{1,2+}$, Wei Liao ${ }^{1 \dagger}$, Qinshui Wu ${ }^{1 \dagger}$, Xiaoshan Huang ${ }^{1 \dagger}$, Zhen Pan ${ }^{1}$, Wang Chen ${ }^{1}$, Shuyi Gu', Zuojun Huang ${ }^{1}$, Yiwen Wang ${ }^{1}$, Xu Tang ${ }^{1}$, Shanshan Liang ${ }^{1}$, Xiaoyan Zhang ${ }^{1}$, Yun Chen ${ }^{1}$, Shuang Chen², Wanying Chen ${ }^{2}$, Yi Jiang ${ }^{3}$, Chen Chen ${ }^{2,4^{*}}$ (I) and Guodong Qiu ${ }^{2,4^{*}}$

\begin{abstract}
Background: Expression of the long non-coding mRNA LINC00152 has been reported to correlate with cancer cell resistance to oxaliplatin (L-OHP). However, little is known regarding the molecular mechanism of LINC00152 in esophageal cancer (EC). Hence, we intended to characterize the role of LINC00152 in EC, with a special focus on epithelial-mesenchymal transition (EMT) and L-OHP resistance.

Methods: We collected EC tissues and identified EC cell lines with higher L-OHP resistance, and then characterized expression patterns of LINC00152, Zeste Homologue 2 (EZH2), Zinc finger e-box binding homeobox (ZEB1) and EMTrelated genes using RT-qPCR and Western blot analysis. Furthermore, their functional significance was identified by gain and loss-of-function experiments. The relationship among LINC00152, EZH2 and ZEB1 was examined using RIP, RNA pull-down and ChIP assays. Additionally, resistance of EC cells to L-OHP was reflected by CCK-8 assay to detect cell viability. Animal experiments were also conducted to detect the effects of the LINC00152/EZH2/ZEB1 on EMT and L-OHP resistance.

Results: LINC00152, EZH2 and ZEB1 were highly expressed in EC tissues and Kyse-150/TE-1 cells. As revealed by assays in vitro and in vivo, LINC00152 positively regulated ZEB1 expression through interaction with EZH2 to enhance EMT and L-OHP resistance in EC cells. In contrast, silencing of LINC00152 contributed to attenuated EMT and drug resistance of EC cells to L-OHP.
\end{abstract}

Conclusions: Our study demonstrates that LINC00152/EZH2/ZEB1 axis can regulate EMT and resistance of EC cells to $\mathrm{L}-\mathrm{OHP}$, thus presenting a potential therapeutic target for EC treatment.

Keywords: Esophageal cancer, LINC00152, Zeste Homologue 2, Zinc finger e-box binding homeobox 1, Epithelialmesenchymal transition, Chemotherapy resistance

*Correspondence: chenchenshantou@126.com; Guodongqiu_dr@163.com †Shuyao Zhang, Wei Liao, Qinshui Wu and Xiaoshan Huang contributed equally to this work

${ }^{2}$ Department of Pharmacology, Shantou University Medical College, Shantou 515000, P.R. China

Full list of author information is available at the end of the article

\section{Background}

Esophageal cancer (EC) ranked 6th in incidence and 7th in cancer-related mortality globally in 2018 [1], and is accompanied by high aggressiveness and low survival rate [2]. Esophageal squamous cell carcinoma (ESCC) accounts for approximately $90 \%$ of global EC cases each

(c) The Author(s) 2020. This article is licensed under a Creative Commons Attribution 4.0 International License, which permits use, sharing, adaptation, distribution and reproduction in any medium or format, as long as you give appropriate credit to the original author(s) and the source, provide a link to the Creative Commons licence, and indicate if changes were made. The images or other third party material in this article are included in the article's Creative Commons licence, unless indicated otherwise in a credit line to the material. If material is not included in the article's Creative Commons licence and your intended use is not permitted by statutory regulation or exceeds the permitted use, you will need to obtain permission directly from the copyright holder. To view a copy of this licence, visit http://creativecommons.org/licenses/by/4.0/. The Creative Commons Public Domain Dedication waiver (http//creativecommons.org/publicdomain/zero/1.0/) applies to the data made available in this article, unless otherwise stated in a credit line to the data. 
year. [3] Current treatment options to improve survival rates of EC patients include radiotherapy [4], surgery, and chemotherapy [5]. Besides, oxaliplatin (L-OHP) is a promising antineoplastic agent that has been increasingly used in EC [6], although L-OHP resistance presents a major medical problem in this form of EC treatment [7]. Previous work has shown that epithelial-mesenchymal transition (EMT) is associated with chemotherapy resistance [8]. Accumulating studies have reported that long non-coding RNAs (lncRNAs) are key regulators of drug resistance and EMT [9-11].

LncRNAs are a class of RNA transcripts of 200 nucleotides in length with limited protein transcriptional potential [12]. However, lncRNAs, are emerging as key regulators of tumor development and progression, which have been studied intensively in gastric cancer, colon cancer [13] and ESCC [14]. Mounting evidence indicates that lncRNAs could serve as targets for EC treatment based on their roles in influencing resistance of cancer cells to chemotherapy, as is notably described for the lncRNAs PART1 [15], DANCR [16] and LINC01419 [17]. Intriguingly, a prior study provided evidence suggesting that LINC00152 (also known as CYTOR) is dysregulated in EC cells, which could position it as a potential biomarker for EC [18]. Moreover, LINC00152 has been implicated to confer L-OHP resistance in colon cancer, highlighting its potential involvement in drug resistance [19]. Therefore, we examine in this study the specific effect of LINC00152 on EMT and EC cell to L-OHP.

Enhancer zeste homolog 2 (EZH2), catalytic subunit of polycomb complex 2 (PRC2), which interacts with LINC00152 in lung adenocarcinoma [20] and the promotion of DNA methylation in ESCC cells by non-coding RNA POU3F3 [21], have drawn much attention in recent years due to their involvement in the development of human diseases, such as [22] lung cancer [23], gastric cancer [24], breast cancer [25], T-cell acute lymphoblastic leukemia [26], and pediatric soft tissue sarcomas [27]. Herein, we hypothesize that LINC00152 might also interact with EZH2 in EC cells.

EMT-induced transcriptional repressor, Zinc finger e-box binding homeobox 1 (ZEB1) is recognized as a stimulator towards imparting an aggressive, metastatic, and therapy-resistant cancer phenotype [28]. In addition, ZEB1 together with EZH2, is reported to play a key role in EMT in respiratory virus infection [29]. Based on our database findings and results from previous studies, we inferred that LINC00152 might regulate ZEB1 via interacting with EZH2 in EC. Therefore, we explored the regulatory relationship of the LINC00152 EZH2/ZEB1 axis and its involvement in EMT as well as resistance of EC cells to L-OHP, aiming to establish a new therapeutic channel for better treatment of EC.

\section{Materials and methods}

Ethics statement

The study protocol was approved by the Ethics Committee and Experimental Animal Ethics Committee of Cancer Hospital of Shantou University Medical College. All individuals signed informed written consent documents. Extensive efforts were made to ensure minimal suffering of the animals used in the study.

\section{Study subjects}

In this study, EC tissues and adjacent normal tissues were collected from 76 EC patients in Cancer Hospital of Shantou University Medical College from 2016 to 2018. None of those patients had received radiotherapy and chemotherapy before surgery.

\section{Cell culture}

The normal human esophageal epithelial cell line Het$1 \mathrm{~A}$ and EC cell lines Kyse-30, Kyse-70, Kyse-150, TE-1 and TE- 6 were purchased from Tumor Cell Bank of the Chinese Academy of Medical Science (Shanghai, China). All these cell lines were cultured in Roswell Park Memorial Institute (RPMI)-1640 medium (61,870,044, Gibco, Carlsbad, CA, USA) containing 10\% fetal bovine serum (FBS, Gibco, Carlsbad, CA, USA), $50 \mathrm{U} / \mathrm{mL}$ penicillin and $50 \mu \mathrm{g} / \mathrm{mL}$ streptomycin $(15,070,063$, Gibco, Carlsbad, CA, USA) in a $37{ }^{\circ} \mathrm{C}$ incubator with $5 \% \mathrm{CO}_{2}$. Oxaliplatin (L-OHP) was dissolved in phosphate buffered saline (PBS) to prepare solutions at different concentrations $\left(0.5,1,2.5,5.0\right.$ and $10.0 \mu \mathrm{M}$, which were stored at $4{ }^{\circ} \mathrm{C}$ until use.

\section{Cell counting kit-8 (CCK-8) assay}

Cell viability was assessed with a CCK-8 kit (GK10001, GLPBIO, Shanghai, China) following the manufacturer's protocol. After adding $100 \mu \mathrm{L}$ of CCK-8 solution in each well, cells were incubated at room temperature for $2 \mathrm{~h}$. The cell viability curve was plotted using optical density (OD) value measured at $460 \mathrm{~nm}$ at each time point. Experiments were independently repeated in triplicate in duplicate.

\section{Transient transfection}

Kyse-150 and TE-1 cells were Three anti-LINC00152 siRNA constructs (named si-LINC00152-1, siLINC00152-2, and si-LINC00152-3), anti-EZH2 siRNA (si-EZH2), anti-ZEB1 siRNA (si-ZEB1), LINC00152 expression vector (oe-LINC00152), EZH2 expression vector (oe-EZH2), ZEB1 expression vector (oe-ZEB1), and their negative controls (NC) were delivered into Kyse-150 and TE-1 cells, respectively, by using Lipofectamine 2000 reagents according to the manufacturer's protocols (Invitrogen, Carlsbad, CA, USA). All siRNA 
Table 1 siRNA sequences

\begin{tabular}{ll}
\hline siRNA & Sequence $\left(5^{\prime}-3^{\prime}\right)$ \\
\hline si-NC & F: 5'-UUCUCCGAACGUGUCACGUTT-3' \\
& R: 5'-ACGUGACACG UUCGGAGAATT-3' \\
si-LINC00152-1 & F: 5'-GGAAUGCAGCUGAAAGAUUTT-3' \\
& R: 5'-AAUCUUUCAGCUGCAUUCCTT-3' \\
si-LINC00152-2 & F: 5'-GGUGGUCUGCCUGUGAUAUTT-3' \\
& R: 5'-AUAUCACAGGCAGACCACCTT-3' \\
Si-LINC00152-3 & F: 5'-TGCCGGATGCAGCTGAAAGATTTCAA-3' \\
& R: 5'-TCGAGAAAAAAGCCGATGGATGCAT-3' \\
Si-EZH2 & F: 5'-AUCAGCUCGUCUGAACCUCUU-3' \\
& R: 5'-AAGAGGUUCAGACGAGCUGAU-3' \\
Si-ZEB1 & F: 5'-CCUAGUCAGCCACCUUUAATT-3' \\
& R: 5'-UUA AAGGUGGCUGACUAGGTT-3'
\end{tabular}

$R T-q P C R$ reverse transcription quantitative polymerase chain reaction, EZH2 Zeste Homologue 2, ZEB1 zinc finger e-box binding homeobox 1 , $F$ forward, $R$ reverse

Table 2 Primer sequences for RT-qPCR

\begin{tabular}{ll}
\hline Gene & Sequence \\
\hline LINC00152 & F: 5'-CTCCAGCACCTCTACCTGTTG-3' \\
& R: 5'-GGACAAGGGATTAAGACACACA-3' \\
EZH2 & F: 5'-GGCTCCTCTAACCATGTTTACAACT-3' \\
& R: 5'-AGCGGTTTTGACACTCTGAACTAC-3' \\
ZEB1 & F: 5'-ACTCTGATTCTACACCGC-3' \\
& R: 5'-TGTCACATTGATAGGGCTT-3' \\
GAPDH & F: 5'-GCACCGTCAAGGCTGAGAAC-3' \\
& R: 5'-ATGGTGGTGAAGACGCCAGT-3' \\
\hline
\end{tabular}

$R T-q P C R$ reverse transcription quantitative polymerase chain

reaction, EZH2 Zeste Homologue 2, ZEB1 zinc finger e-box binding homeobox 1, GAPDH glyceraldehyde-3-phosphate dehydrogenase, $F$ forward, $R$ reverse

constructs and expression vectors were purchased from Shanghai Sangon Biotech company (Shanghai, China), who also generated primer sequences and plasmid construction for siRNA sequences, as shown in Table $1.48 \mathrm{~h}$ after transfection, cells were collected for further analysis. The experiment was repeated in triplicate.

\section{Reverse transcription quantitative polymerase chain reaction ( $R T-q P C R)$}

Total RNA from tissues or cells was extracted using Trizol reagent (Thermo Fisher Scientific, Waltham, MA, USA). The synthesis of primer was conducted by Sangon Biotech Co., Ltd, (Shanghai, China) (Table 2). Reverse transcription was performed according to instructions of High-Capacity cDNA Reverse Transcription Kit $(4,368,813$, Thermo Fisher Scientific, Waltham, MA, USA) to generate cDNA. Real-Time Quantitative fluorogenic PCR assay was developed according to instructions of PCR kit $(11,732,020$, Thermo Fisher Scientific, Waltham, MA, USA). The relative expression level of target genes was measured by $2^{-\Delta \Delta \mathrm{Ct}}$ method normalized to glyceraldehyde-3-phosphate dehydrogenase (GAPDH) mRNA expression.

\section{RNA immunoprecipitation (RIP) assay}

The binding of LINC00152 to EZH2 protein was measured using a RIP kit (17-701, Millipore Corp, Billerica, MA, USA) according to the manufacturer's instructions. Upon cell confluence in six-well plate reaching about 80-90\%, cells in each well were lysed with an equal volume of RIPA lysis buffer (P0013B, Beyotime Institute of Biotechnology, Shanghai, China) for $5 \mathrm{~min}$ in an ice-bath and then centrifuged at $14,000 \mathrm{rpm}$ for $10 \mathrm{~min}$ at $4{ }^{\circ} \mathrm{C}$. A certain portion of supernatant was removed to serve as an input, and the remainder was co-precipitated by incubation with antibody. In brief, $50 \mu \mathrm{L}$ of magnetic beads in each coprecipitation reaction system were resuspended in $100 \mu \mathrm{L}$ of RIP wash buffer and incubated with $5 \mu \mathrm{g}$ of corresponding antibody. Subsequently, the magnetic bead-antibody complex was washed and resuspended in $900 \mu \mathrm{L}$ of RIP wash buffer and then incubated with 100 $\mu \mathrm{L}$ of cell extract overnight at $4{ }^{\circ} \mathrm{C}$, whereupon it was placed on a magnetic stand to collect the magnetic beadprotein complex. Afterwards, samples and inputs were separately treated with proteinase $\mathrm{K}$ to extract RNA for subsequent PCR detection of LINC00152. The antibodies used for RIP were EZH2 (1:100, ab186006, Abcam, Cambridge, UK), SUZ12 (1:100, ab12073, Abcam, Cambridge, UK) and EED (SC-293,203, 1:100, Santa Cruz Biotechnology, Santa Cruz, CA, USA) with IgG (1:100, ab200699, Abcam, Cambridge, UK) as a NC. The experiment was repeated in triplicate.

\section{RNA pull-down}

Cells were transfected with biotinylated LINC00152Sense strand and LINC00152-Antisense strand (50 nM for each). At $48 \mathrm{~h}$ post transfection, cells were washed, vortexed and incubated with specific cell lysis buffer (Ambion, Austin, TX, USA) for $10 \mathrm{~min}$, taking a $50 \mathrm{~mL}$ portion of cell lysate as a control. The remaining lysate was incubated with M-280 streptavidin magnetic beads (Sigma, St. Louis, MO, USA) pre-coated with RNase-free and yeast tRNA (Sigma, St. Louis, MO, USA) for $3 \mathrm{~h}$ at $4{ }^{\circ} \mathrm{C}$, followed by two washes with cold lysis buffer, three washes with low salt buffer, and one wash with high salt buffer. Afterwards, total protein was extracted with highefficiency RIPA lysis buffer and then the expression of EZH2 (1:100, ab186006, Abcam, Cambridge, UK) was determined by Western blot analysis. The experiment was repeated in triplicate. 


\section{Fluorescence in situ hybridization (FISH)}

The localization of LINC00152 in cells was examined using a FISH Kit (F32956, Thermo Fisher Scientific, Waltham, MA, USA) with digoxigenin-labeled LINC00152 probe, purchased from Sigma-Aldrich, St. Louis, MO, USA. Cell nuclei were stained with 4', 6-diamidino-2-phenylindole (DAPI, D9542, SigmaAldrich, St. Louis, MO, USA) for $10 \mathrm{~min}$. A laser confocal scanning microscope (FV1000, Olympus, Tokyo, Japan) was employed to take photos of fluorescence staining, and experiment was repeated in triplicate.

\section{Western blot analysis}

Total protein was extracted using a protein extraction kit (BC3640, Beijing Solarbio Science and Technology Co., Ltd., Beijing, China), and was then quantified using a bicinchoninic acid (BCA) protein assay kit (20201ES76, Yeasen Biotech Co., Ltd., Shanghai, China). The extracted proteins were separated by $10 \%$ sodium dodecyl sulfatepolyacrylamide gel electrophoresis (SDS-PAGE), and then transferred onto a polyvinylidene fluoride (PVDF; Bio-Rad Laboratories, Hercules, CA, USA) membrane. Subsequently, the PVDF membrane were incubated overnight at $4{ }^{\circ} \mathrm{C}$ with diluted primary monoclonal antibodies against the target proteins GAPDH (ab181602, 1:5000), E-cadherin (ab1416, 1:1000), vimentin (ab193555, $1: 1000)$, cleaved poly adenosine diphosphate-ribose polymerase (PARP; ab32064, 1:1000), cleaved Caspase3 (ab2302, 1:1000), EZH2 (ab186006, 1:1000), ZEB1 (ab228986, 1:1000), and PARP. Afterwards, the membrane was blotted with corresponding secondary antibody conjugated to horseradish peroxidase, namely, goat anti-rabbit antibody to immunoglobulin G (IgG; ab6721, 1:2000) or rabbit anti-mouse (ab6728, 1:2000) for $1 \mathrm{~h}$ at $4{ }^{\circ} \mathrm{C}$. All antibodies were purchased from Abcam (Cambridge, UK). After washed with PBS with 5 min for each time, membranes were incubated with enhanced chemiluminescence (ECL) (Pierce, Waltham, MA, USA) for about $1 \mathrm{~min}$ and were then semi-quantified using an Image Quant LAS-4000 image reader (GE Healthcare, Piscataway, NJ, USA). The analysis of protein expression levels was obtained as the ratio of gray values between proteins of interest and internal reference standard GAPDH.

\section{Chromatin immunoprecipitation (ChIP)}

ChIP experiments were performed using EZ-Magna ChIP kit (EMD Millipore Corp., Billerica, MA, USA) according to protocol provided by manufacturer. MSCs cells were fixed with $1 \%$ paraformaldehyde and incubated with glycine for $10 \mathrm{~min}$ to crosslink DNA and protein. Then, cells were lysed with lysis buffer and sonicated to produce 200-300 bp chromatin fragments. Next, lysate was immunoprecipitated with magnetic protein A beads, which were bound to antibodies targeting the proteins of interest, namely, EZH2 (ab191250, 1:20, Abcam, Cambridge, UK) and methylation of lysine 27 on histone H3 (H3K27me3; ab6002, 1:20, Abcam, Cambridge, UK). Meanwhile, NC was added with IgG (ab2410, 1:20, Abcam, Cambridge, UK). Finally, precipitated ZEB1 was analyzed by RT-qPCR assay. ZEB CHIP primer was (F: 5'-AGGCGTGGGACTGATGGTAG-3'， R: 5'-ATTCTC CCTGTACCCTGTGC-3').

\section{Xenograft tumor in nude mice}

Twenty-four specific pathogen-free (SPF) female BALB/C nude mice aged 4 weeks and weighing 18-22 g purchased from Shanghai SLAC Laboratory Animal Co., Ltd. (Shanghai, China) were employed for animal experiments. EC cells Kyse-150 were cultured in a culture dish. When cell confluence reached $90 \%$, cells were detached with a conventional trypsin buffer and rinsed twice with Dulbecco's modified Eagle's medium (DMEM) to prepare a single cell suspension. The cell suspension was centrifuged at $1500 \mathrm{rpm}$ for $3 \mathrm{~min}$ to remove the supernatant, followed by addition of DMEM. The number of blood cells was counted under the microscope and cell concentration was adjusted to $5 \times 10^{7}$ cells $/ \mathrm{mL}$. Cells were preserved on ice for $1 \mathrm{~h}$ and then inoculated subcutaneously into the nude mice. About 10 days later, solid tumors had formed; upon attaining a tumor volume of about $100 \mathrm{~mm}^{3}$, groups of mice were injected intravenously with (1) oe-NC and si-NC, (2) oe-NC and si-NC plus L-OHP, (3) oe-LINC0015 and si-NC plus L-OHP, or (4) both oe-LINC0015 and si-ZEB1 plus L-OHP to explore how LINC00152 regulates the EZH2/ZEB1 axis in the context of EC cell drug resistance. 25 days after treatment, the mice were euthanized, and the tumors were extracted from the mice, washed in normal saline, and photographed.

\section{Statistical analysis}

The data were processed using SPSS 21.0 statistical software (IBM Corp, Armonk, NY, USA). Measurement data were expressed as mean \pm standard deviation. Paired data in compliance with normal distribution and homogeneity between cancer tissues and adjacent tissues were compared using a paired $t$-test. Comparisons among multiple groups were conducted by one-way analysis of variance (ANOVA) with Tukey's post hoc test. Data at different time points and different concentrations were compared by repeated measures ANOVA. A value of $p<0.05$ indicated significant difference. 


\section{Results}

LINC00152 is highly expressed in EC tissues and cells and enhances EC cell resistance to L-OHP

It has been reported that LINC00152 is highly expressed in EC, which might induce the occurrence of EC [18]. Besides, LINC00152 contributes to colon cancer resistance to L-OHP [19]. We now sought to investigate whether LINC00152 augmented EC cells to develop L-OHP resistance. First, the findings of the RT-qPCR assay indicated notably increased expression level of LINC00152 in EC tissues compared to normal adjacent tissues (Fig. 1a; $p<0.05$ ). Accordingly, compared with Het-1A cell line, the expression level of LINC00152 was notably elevated in Kyse-30, Kyse-70, Kyse-150, TE-1 and TE-6 cell lines, among which, expression of LINC00152 was highest in Kyse-150 and TE-1 cell lines (Fig. 1b; $p<0.05)$.

The resistance to L-OHP was then tested among the five different EC cell lines. Firs, different doses of L-OHP $(0.0,0.5,1.0,2.5,5.0,10.0 \mu \mathrm{M})$ was added to theseEC cell lines followed by culture for $72 \mathrm{~h}$. CCK-8 assay results indicated a L-OHP dose-dependent decrease of survival rate of all cells, with survival rate of Kyse-150 and TE-1 cells higher than those of other 3 cells at 1.0, 2.5, 5.0, and $10 \mu \mathrm{M}$ L-OHP concentrations (Fig. 1c; $p<0.05$ ). Next, the $5 \mathrm{EC}$ cell cultures were all treated with $10 \mu \mathrm{M}$ L-OHP and their cell viability and expression of LINC00152 were detected by CCK- 8 and RT-qPCR assay, respectively, at $0,24,48$, and 72 hours in culture. Again, the aforementioned data illustrated that Kyse-150 and TE-1 cells had higher survival rate than other three cell lines at 48 and $72 \mathrm{~h}$ of treatment (Fig. 1d; $p<0.05$ ). Meanwhile, expression of LINC00152 was notably elevated in Kyse-150 and TE-1 cells with treatment for 48 and 72 h (Fig. 1e; $p<0.05)$.

To detect resistance to L-OHP and EMT of different EC cell lines, different EC cell lines were treated with $10 \mu \mathrm{L}-\mathrm{OHP}$ for $72 \mathrm{~h}$. Western blot analysis suggested that, compared with cells treated with equal PBS, protein expression of cleaved PARP, cleaved Caspase 3 and E-cadherin was notably elevated in the Kyse-30, Kyse-70, TE-6, Kyse-150 and TE-1 cells, which were resistant to L-OHP $(p<0.05)$, while expression of vimentin protein notably diminished $(p<0.05)$. Compared with L-OHPresistant Kyse-30, Kyse-70, TE-6 cells, expression of cleaved PARP, cleaved Caspase 3 and E-cadherin protein were diminished in L-OHP-resistant Kyse-150 and TE-1 cells $(p<0.05)$, and expression of vimentin protein elevated (Fig. $1 \mathrm{f}-\mathrm{i} ; p<0.05$ ).

These results indicated that different EC cell lines exhibited different degrees of sensitivity to L-OHP as evidenced by their cell survival rates, and that the lines with
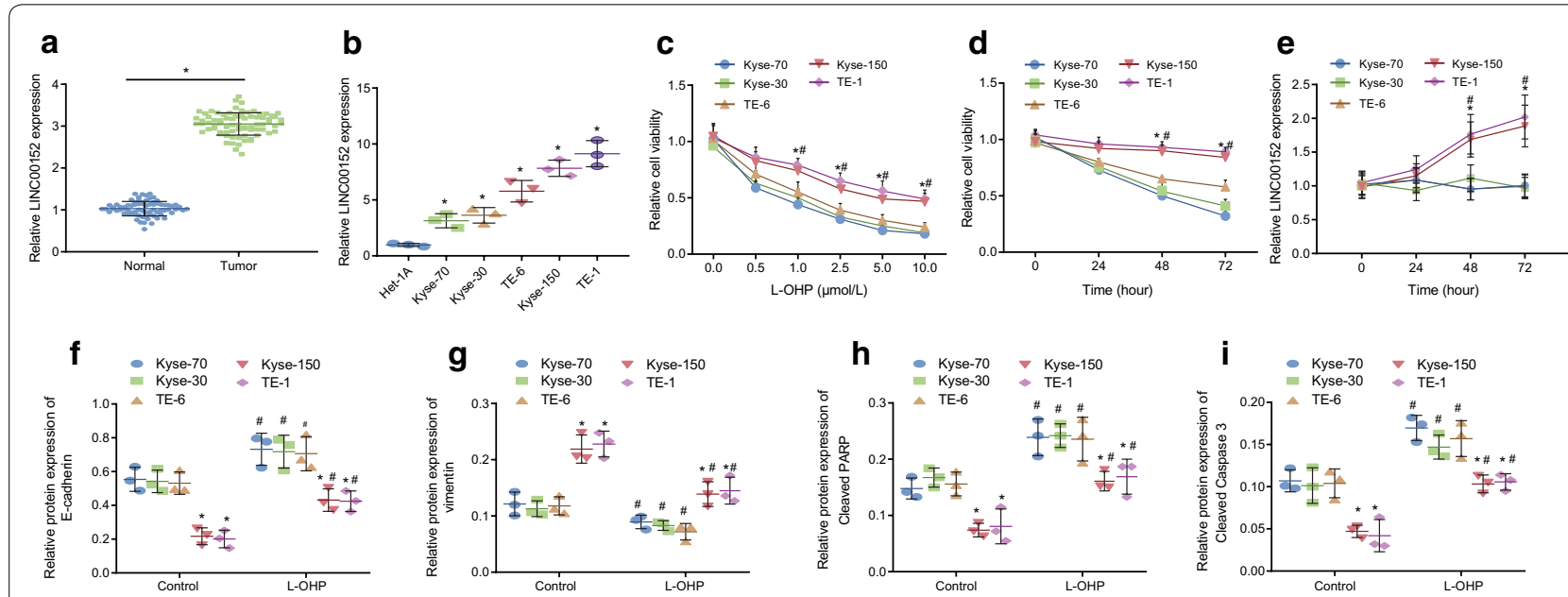

Fig. 1 upregulated LINC00152 is associated with EC cell resistance to L-OHP. a the expression of LINC00152 in EC tissues and normal adjacent tissues detected by RT-qPCR assay. * $p<0.05$ vs. normal adjacent tissues (analyzed by paired $t$-test). $\mathbf{b}$ the expression of LINC00152 in Kyse-30, Kyse-70, Kyse-150, TE-1 and TE-6 cell lines detected by RT-qPCR assay. ${ }^{*} p<0.05$ vs. Het-1A cells (analyzed by one-way ANOVA with Tukey's post hoc test). $\mathbf{c}$ cell survival rate after $72 \mathrm{~h}$ of treatment with different doses of L-OHP $(0,0.5,1.0,2.5,5.0,10.0 \mu \mathrm{M})$ detected by CCK-8 assay. $\mathbf{d}$ cell survival rate after 0, 24, 48 and $72 \mathrm{~h}$ of treatment with $10.0 \mu \mathrm{M}$ of L-OHP detected by CCK-8 assay. e, the expression of LINC00152 after 0, 24, 48 and $72 \mathrm{~h}$ of treatment with $10.0 \mathrm{\mu M}$ of L-OHP detected by RT-qPCR assay. * indicates $p<0.05$ when Kyse-150 vs.Kyse-30, Kyse-70, and TE-6, respectively; \# $p<0.05$ when TE-1 vs. Kyse-30, Kyse-70, and TE-6, respectively (analyzed by one-way ANOVA with Tukey's post hoc test or repeated measures ANOVA with Bonferroni corrections). $\mathbf{f}-\mathbf{i}$, Western blot analysis of E-cadherin, vimentin, cleaved PARP, and cleaved Caspase 3 in five EC cells with or without after 72-hour treatment of $10.0 \mu \mathrm{M} \mathrm{L-OHP.} \mathrm{*} \mathrm{indicates} p<0.05$ vs. parental or L-OHP-treated Kyse-30, Kyse-70, and TE-6, respectively; \# indicates $p<0.05$ vs. corresponding parental EC cells (analyzed by one-way ANOVA with Tukey's post hoc test or repeated measures ANOVA with Bonferroni corrections). Data are presented as mean \pm standard deviation of three technical replicates 
highest resistance (Kyse-150 and TE-1 cell lines) had the highest expression of LINC00152 and could affect EMT. Due to their higher drug resistance to L-OHP, Kyse150 and TE-1 cell lines were selected for subsequent experiments.

\section{Downregulation of LINC00152 reduces EMT and EC cell resistance to $L-O H P$}

The aforementioned evidence shows that LINC00152 might be a factor in EMT and resistance to L-OHP in EC cells. To verify effect of LINC00152 on EC cells, we overexpressed and knocked down LINC00152 separately in Kyse-150 and TE-1 cells. Results of RT-qPCR revealed that compared with si-NC, all anti-LINC00152 siRNA constructs (si-LINC00152-1, si-LINC00152-2, and siLINC00152-3) effectively knocked down LINC00152. As shown in Fig. 2a, si-LINC00152-1 was selected to knockdown LINC00152-1 for subsequent experiments. Likewise, compared with oe-NC, expression level of LINC00152 in oe-LINC00152 group was notably elevated (Fig. 2b; $p<0.05$ ). After LINC00152 was overexpressed or knocked down in Kyse-150 and TE-1 cells, the cells were treated with different doses of L-OHP for 72 h. CCK-8 assay then showed that, compared with cells transfected with oe-NC, the survival rate of oe-LINC00152 transfected cells was notably elevated $(p<0.05)$. Accordingly, compared with si-NC, survival rate of si-LINC001521 -treated cells was notably diminished at doses of 1.0, 2.5, 5.0, and $10 \mu \mathrm{M}$ (Fig. $2 \mathrm{c}-\mathrm{d} ; p<0.05$ ). Setting the dose of L-OHP treatment to $10 \mu \mathrm{M}$, the CCK- 8 assay suggested that, compared with cells transfected with oe-NC, survival rate in oe-LINC00152 notably elevated at 24, 48 and $72 \mathrm{~h}(p<0.05)$, while compared with si-NC treatment, survival rate following si-LINC00152-1 treatment notably diminished (Fig. 2e-f; $p<0.05$ ).

Next, we investigated whether downregulation of LINC00152 could affect EMT and drug resistance of EC cells. After overexpressing or knocking down LINC00152 in Kyse-150 and TE- 1 cells, these cells were treated with $10 \mu \mathrm{M} \mathrm{L}-\mathrm{OHP}$ for $72 \mathrm{~h}$. Results of Western blot analysis suggested that after the L-OHP treatment, there was notably elevated protein expression of E-cadherin, cleaved PARP and cleaved Caspase 3 in si-LINC00152-1 transfected cells compared with the si-NC group $(p<0.05)$, while expression of vimentin markedly diminished $(p<0.05)$. On the other hand, compared with cells transfected with oe-NC, expression level of E-cadherin, cleaved PARP and cleaved Caspase 3 protein was notably lower in oe-LINC00152 transfected EC cells $(p<0.05)$, while expression of vimentin was notably elevated (Fig. 2g-n; $p<0.05$ ). These results suggested that downregulation of LINC00152 was able to decrease EMT and drug resistance in EC cells.

\section{LINC00152 promotes EC cell resistance to L-OHP and EMT through recruitment of EZH2}

A previous study suggested that LINC00152 can interact with EZH2 to regulate expression of their downstream genes [20]. Here, FISH assay results suggested LINC00152 was expressed and localized in the nucleus and cytoplasm in Kyse-150 and TE-1 cells (Fig. 3a). It was also previously reported that lncRNA MALAT1 releases EZH2 by binding to the PRC2 complex, a main component of which is EZH2, which hinders transcriptional inhibition of downstream genes, thereby promoting expression of downstream genes [30]. Thus, we inferred that LINC00152 would release EZH2 by binding to a complex of PRC2, thus reducing trimethylation of h3k27 and blocking transcriptional inhibition of the zeb1 gene, consequently promoting the expression of zeb1. to verify whether linc00152 bound to prc2, interactions between linc00152 and ezh2, suz12 along with eed were assessed by rip assay. analysis of rt-qpcr revealed that the amounts of linc00152 pulled by ezh2, sUZ12 and EED were notably higher than with IgG (Fig. 3b-c; $p<0.05$ ). Next, RNA pull-down assay was used to verify further an interaction between LINC00152 and EZH2. The sense and antisense chains of LINC00152 were incubated with cell extracts, and the EZH2 protein content was measured by Western blot analysis. The aforementioned data illustrated that EZH2 protein interacted with the sense chain of LINC00152 (Fig. 3d). Next, we investigated whether EZH2 mediated effects of LINC00152 on the regulation of EMT and drug resistance of EC cells. First, RT-qPCR results displayed that mRNA expression of EZH2 in EC tissues, Kyse-150 and TE-1 cells were notably higher than those in normal adjacent tissues and Het-1A cells (Fig. 3e-f; $p<0.05$ ). Then, results of Western blot analysis suggested that protein expression of EZH2 in EC tissues, Kyse-150 and TE-1 cells was also notably higher than that in normal adjacent tissues and Het-1A, respectively (Fig. 3g-h; $p<0.05$ ).

Results of the CCK- 8 assay suggested that, compared with cells transfected with oe-LINC00152, survival rate in cells transfected with oe-LINC00152 and si-EZH2 was notably lower after treatments of different doses of L-OHP in Kyse-150 and TE-1 cells for $72 \mathrm{~h}(p<0.05)$. Compared with cells transfected with oe-LINC00152 and si-EZH2, survival rate of cells transfected with siEZH2 was also notably lower (Fig. 3i-j; $p<0.05$ ). Moreover, Western blot analysis suggested that after $10 \mu \mathrm{M}$ $\mathrm{L}-\mathrm{OHP}$ treatment for $72 \mathrm{~h}$, protein expression of E-cadherin and cleaved PARP/PARP and cleaved Caspase 3/ Caspase 3 in cells transfected with oe-LINC00152 and si-EZH2 was notably elevated compared with that in oe-LINC00152 transfected-cells $(p<0.05)$, while protein expression of vimentin was notably diminished 

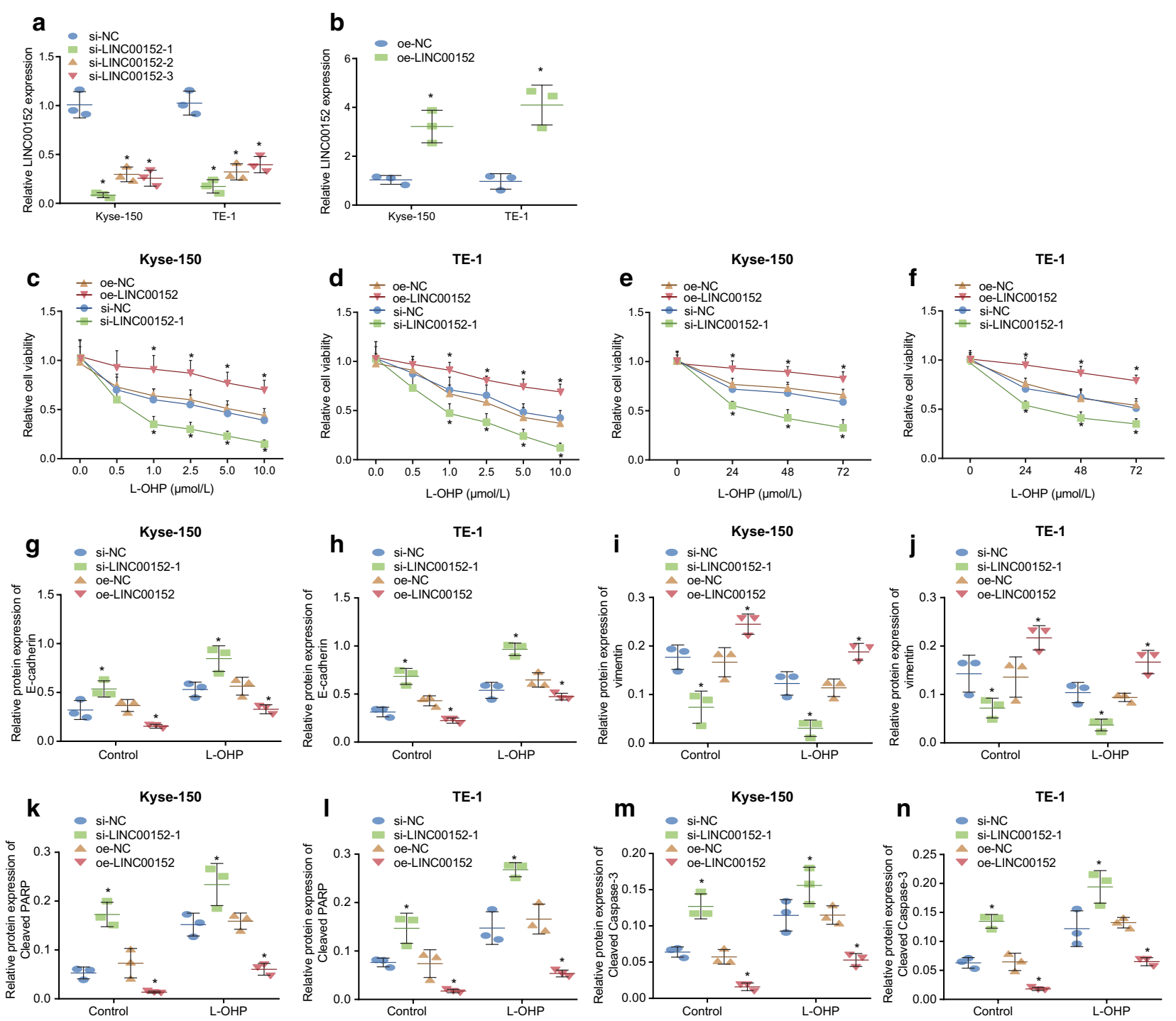

Fig. 2 Silencing of LINC00152 attenuated EMT and EC cell resistance to L-OHP. a-b the expression of LINC00152 after overexpressing or knocking down LINC00152 in Kyse-150 and TE-1 cells detected by RT-qPCR assay. c-d cell survival rate after $72 \mathrm{~h}$ of treatment with different concentrations of L-OHP $(0,0.5,1.0,2.5,5.0,10.0 \mu \mathrm{M})$ in Kyse-150 and TE-1 cells detected by CCK-8 assay. e-f cell survival rate after 0, 24, 48 and $72 \mathrm{~h}$ of treatment with $10.0 \mu \mathrm{M}$ of L-OHP in Kyse-150 and TE-1 cells detected by CCK-8 assay. $\mathbf{g}-\mathbf{n}$ the protein expression of E-cadherin, cleaved PARP and cleaved Caspase 3 in Kyse-150 and TE-1 cells after $72 \mathrm{~h}$ of treatment with $10.0 \mu \mathrm{mol} / \mathrm{L}$ of L-OHP determined by Western blot analysis. ${ }^{*} p<0.05 \mathrm{vs}$. the oe-NC group or the si-NC group. Data in compliance with normal distribution and homogeneity were compared using unpaired $t$-test. Comparisons among multiple groups were conducted by ANOVA with Tukey's post hoc test. Data at different time points and different concentrations were compared by repeated measures ANOVA. Data are presented as mean \pm standard deviation of three technical replicates

$(p<0.05)$. Compared with those in oe-LINC00152 and si-EZH2 transfected-cells, protein expression of E-cadherin, cleaved PARP/PARP and cleaved Caspase $3 /$ Caspase 3 in si-EZH2 transfected-cells was notably enhanced $(p<0.05)$, while protein expression of vimentin was notably diminished (Fig. $3 \mathrm{k}-\mathrm{l})(p<0.05)$. The above results indicated that LINC00152 upregulates EMT and drug resistance through positively regulating EZH2.

\section{LINC00152 increases expression of ZEB1 through EZH2 in EC cells}

EZH2 was highly expressed in EC tissues as well as Kyse150 and TE- 1 cell lines. It has been reported that overexpression of EZH2 can promote expression of ZEB1 [31]. Here, we analyzed the expression level of ZEB1 in EC tissues, Kyse-150 and TE-1 cells by RT-qPCR (Fig. 4a-b) and Western blot analysis (Fig. 4c-d). The results illustrated that mRNA level and protein expression level of 


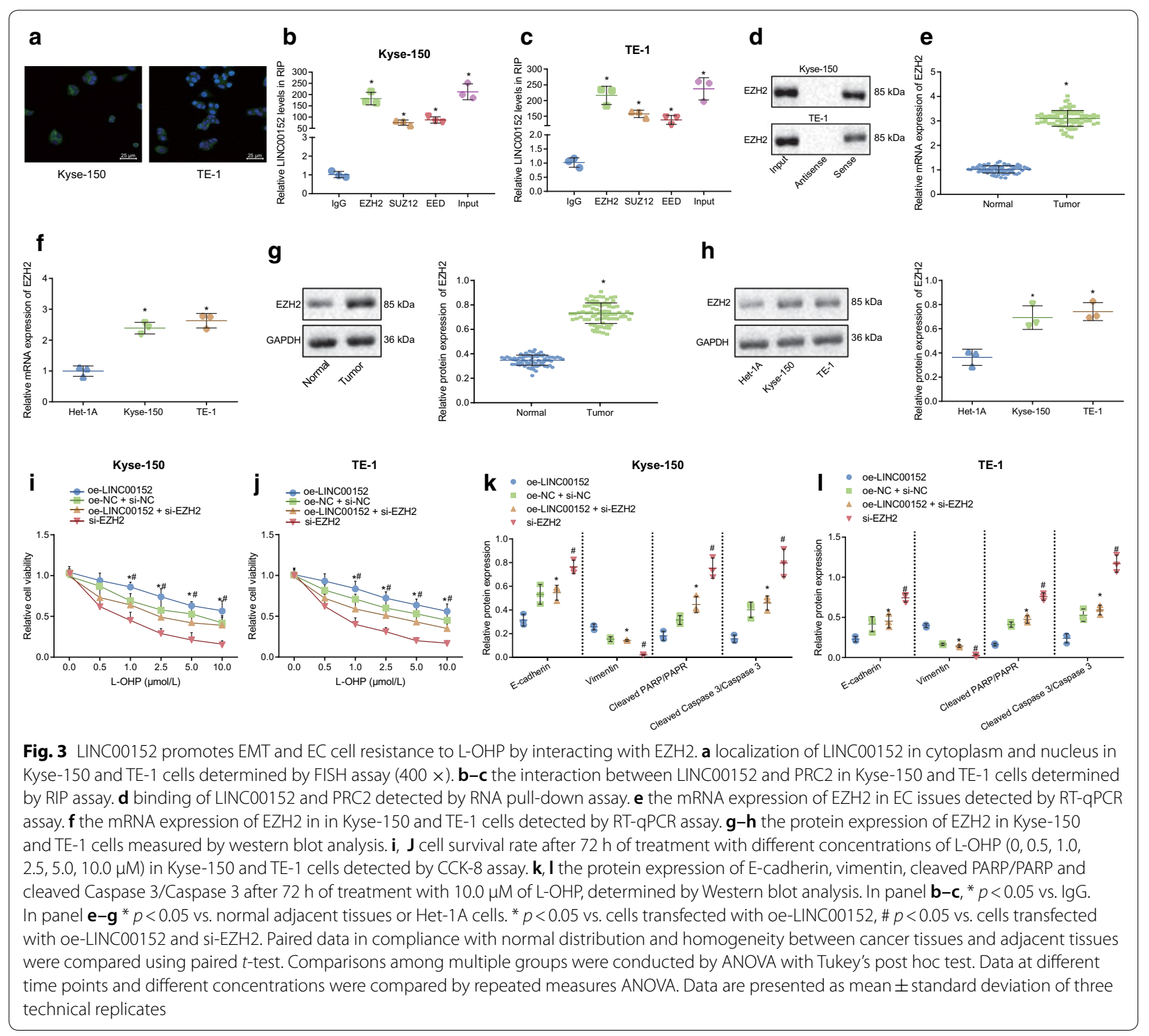

ZEB1 in EC tissues, Kyse-150 and TE-1 cells were notably higher than those in normal adjacent tissues and Het-1A cells $(p<0.05)$.

In addition, we tested whether EZH2 could also increase expression of ZEB1 in EC cells. Results of ChIP assay suggested that treatment with $10.0 \mu \mathrm{M} \mathrm{L-OHP}$ for $72 \mathrm{~h}$, there was significant enrichment of EZH2 and $\mathrm{H} 3 \mathrm{k} 27 \mathrm{me} 3$ in the promoter region of ZEB1 gene in siLINC00152-1 transfected Kyse-150 and TE-1 cells compared with si-NC transfected-cells (Fig. 4e-f; $p<0.05$ ). After different transfections of Kyse-150 and TE- 1 cells, results of RT-qPCR assay (Fig. $4 \mathrm{~g}-\mathrm{h}$ ) and Western blot analysis (Fig. 4i-k) displayed that $\mathrm{mRNA}$ and protein expression of ZEB1 in si-EZH2 and si-LINC00152-1 transfected-cells were notably lower than those in oe-NC and si-NC transfected-cells $(p<0.05)$. On the other hand, mRNA and protein expression of ZEB1 in oe-EZH2 and oe-LINC00152 transfected-cells were notably higher. Compared with oe-LINC00152 transfected-cells, mRNA and protein expression of ZEB1 were notably diminished in oe-LINC00152 and si-EZH2 transfected cells. All these results demonstrated that EZH2 upregulated expression of ZEB1 in EC cells.

Further, we tested whether downregulation of ZEB1 could delay EMT and drug resistance of EC cells to L-OHP. Results of RT-qPCR (Fig. 4l) and Western blot analysis (Fig. 4m-n) suggested mRNA and protein expression of ZEB1 in si-ZEB1 transfected-cells 


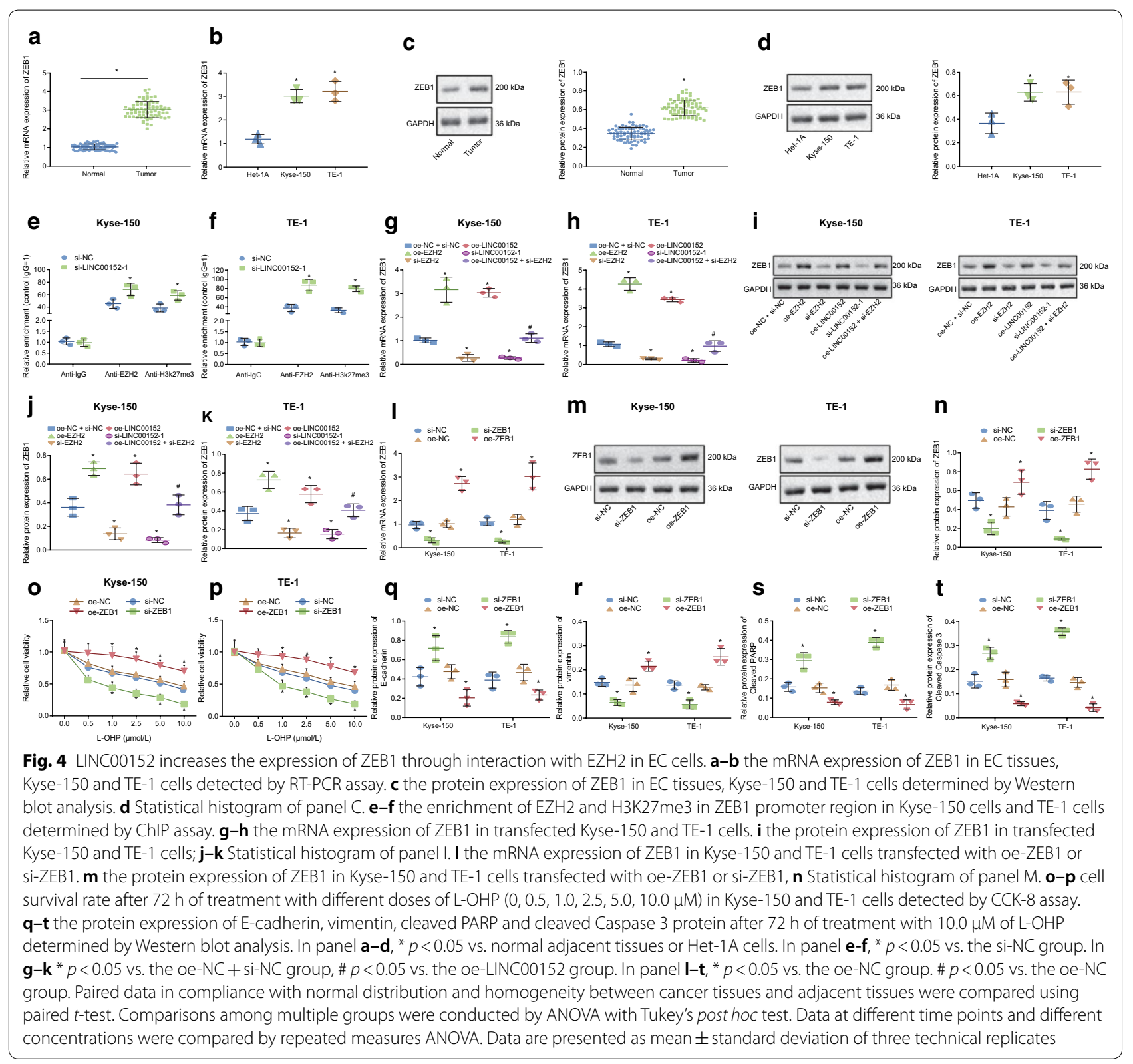

was notably lower than that in si-NC transfected-cells $(p<0.05)$. Conversely, mRNA and protein expression of ZEB1 in oe-ZEB1 transfected-cells was notably higher than in oe-NC transfected-cells. The CCK- 8 assay results suggested that, after treatment with different doses of L-OHP for $72 \mathrm{~h}$, the survival rate in oe-ZEB1 transfected Kyse-150 and TE-1 cells was notably elevated compared with oe-NC transfected cells $(p<0.05)$, and notably diminished in si-ZEB1 transfected-cells compared with si-NC transfected-cells (Fig. 4o-p) $(p<0.05)$. Then, we determined expression of E-cadherin, vimentin, cleaved PARP and cleaved Caspase 3 in Kyse-150 and TE-1 cells treated with $10 \mu \mathrm{M}$ L-OHP for $72 \mathrm{~h}$. Results indicated that, compared with si-NC transfected cells, protein expression of E-cadherin, cleaved PARP and cleaved Caspase 3 in si-ZEB1 transfected-cells was notably elevated $(p<0.05)$, and vimentin expression was notably diminished. On the other hand, compared with oe-NC transfected-cells, protein expression of E-cadherin, cleaved PARP and cleaved Caspase 3 was notably diminished, and vimentin expression was notably elevated (Fig. $4 \mathrm{q}-\mathrm{t} ; p<0.05$ ). The above results indicated that LINC00152 upregulated expression of ZEB1 through itsinteraction with EZH2, thus promoting EMT and drug resistance of EC cells to L-OHP. 


\section{Downregulation of ZEB1 reverses promotive effect of LINC00152 on EMT and EC cell resistance to L-OHP}

To verify further the effects of LINC00152 on regulation of EMT and drug resistance in EC cells was obtained through ZEB1, we transfected Kyse-150 and TE-1 cells with corresponding plasmids and then treated them with different doses of L-OHP for $72 \mathrm{~h}$. The findings of the CCK-8 assay indicated a notably lower survival rate in oe-LINC00152 and si-ZEB1 transfected cells compared with oe-LINC00152 transfected cells, and that survival rate in si-ZEB1 transfected-cells was notably diminished compared with oe-LINC00152 and si-ZEB1 transfected cells (Fig. 5a-b). Then, findings of Western blot analysis for cells treated with $10 \mu \mathrm{M} \mathrm{L}-\mathrm{OHP}$ for $72 \mathrm{~h}$ displayed that, compared with oe-LINC00152 transfected cells, protein expression of E-cadherin, cleaved PARP/PARP and cleaved Caspase 3/Caspase 3 in oe-LINC00152 and si-ZEB1 transfected cells was notably elevated, while vimentin was notably diminished. In comparison with oe-LINC00152 and si-ZEB1 transfected-cells, protein expression of E-cadherin, cleaved PARP/PARP and cleaved Caspase 3/Caspase 3 in si-ZEB1 transfected cells was notably elevated, and vimentin was notably attenuated (Fig. $5 \mathrm{c}-\mathrm{d}$ ). These results suggested that high expression of LINC00152 in EC cells promotes EMT and resistance of EC cells to L-OHP by upregulating ZEB1.

\section{LINC00152 enhances EMT and EC cell resistance to L-OHP by regulating the EZH2/ZEB1 axis in vivo}

A xenograft tumor model in nude mice was established to probe further how LINC00152 could promote EMT and resistance of EC cells to L-OHP treatment via the EZH2/ZEB1 axis. EC cells were subcutaneously inoculated into the nude mice to construct the EC tumor model, and the tumor-bearing mice were further treated with L-OHP combined with oe-LINC00152 or si-ZEB1. Results showed that, the tumor growth rate was increased in response to treatments with oe-LINC00152 plus L-OHP versus the treatments with oe-NC and si-NC plus L-OHP, thus revealing that overexpressed LINC00152 could elevate the resistance of EC. Furthermore, the treatments with si-ZEB1 plus L-OHP resulted in reduced tumor growth rate, indicating that ZEB1 knockdown could effectively attenuate the development of EC. Additionally, in comparison with the treatment of oe-LINC00152 and si-NC plus L-OHP, the treatment of oe-LINC00152 and si-ZEB1 plus L-OHP led to decreased tumor growth rate, indicating that silencing ZEB1 could inhibit the resistance in EC induced by LINC00152 overexpression (Fig. 6a) $(p<0.05)$.

RT-qPCR was conducted to measure the relative expression of LINC00152, EZH2, and ZEB1 while Western blot analysis was carried out to detect the protein expression of EZH2, and ZEB1. The results showed that the expression of LINC00152, EZH2, and ZEB1 was elevated in the tumors of mice treated with oe-LINC00152 and si-NC plus L-OHP versus the mice treated with oe-NC and si-NC plus L-OHP. The treatment with oe-LINC00152 and si-ZEB1 plus L-OHP decreased LINC00152, EZH2, and ZEB1 expression compared to the treatment with oe-LINC00152 and si-NC plus L-OHP (Fig. 6b, c) $(p<0.05)$.

These results revealed that, in the EC xenograft tumor model, treatment with oe-LINC00152 alone could induce greater drug resistance of EC cells, while the combination of oe-LINC00152 and si-ZEB1 increase the sensitivity of
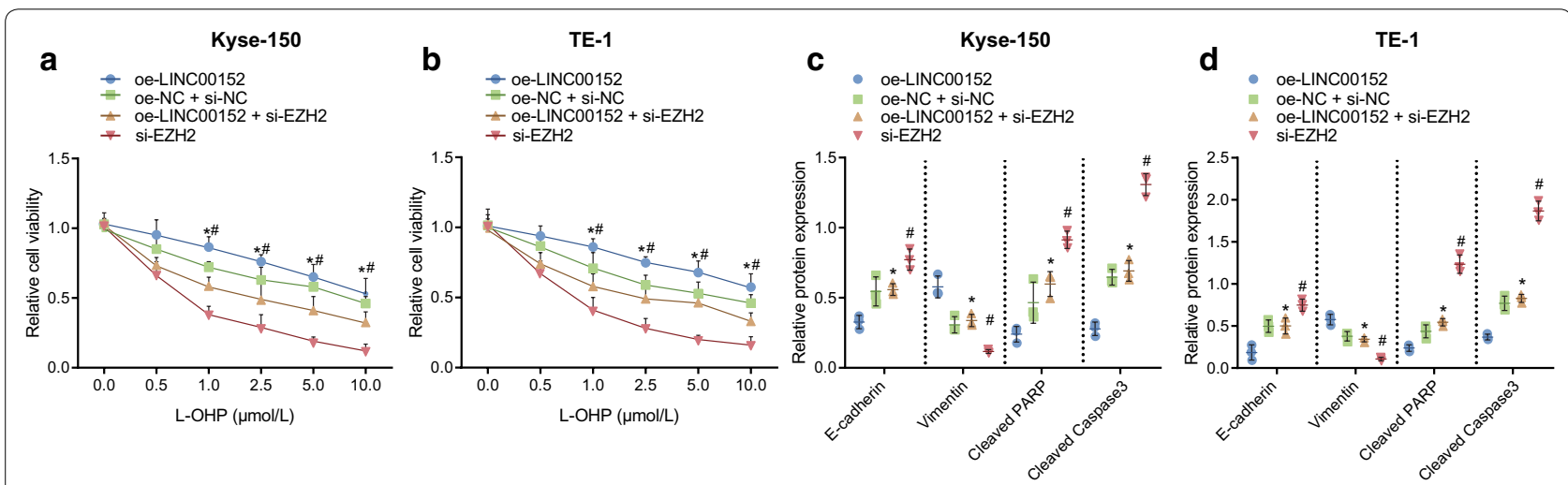

Fig. 5 LINC00152 promotes EMT and EC cell resistance to L-OHP by upregulating ZEB1. a-b cell survival rate after $72 \mathrm{~h}$ of treatment with different concentrations of L-OHP $(0,0.5,1.0,2.5,5.0,10.0 \mu \mathrm{M})$ in Kyse-150 and TE-1 cells detected by CCK-8 assay. $\mathbf{c}-\mathbf{d}$, the expression of E-cadherin, vimentin, cleaved PARP/PARP and cleaved Caspase 3/Caspase 3 proteins in Kyse-150 (c) and TE-1 cells (d) after $72 \mathrm{~h}$ treatment with $10.0 \mu \mathrm{M}$ of L-OHP determined by Western blot analysis. \# $p<0.05$ vs. the si-ZEB1 group. Comparisons among multiple groups were conducted by ANOVA with Tukey's post hoc test. Data at different time points and different concentrations were compared by repeated measures ANOVA. Data are presented as mean \pm standard deviation of three technical replicates 

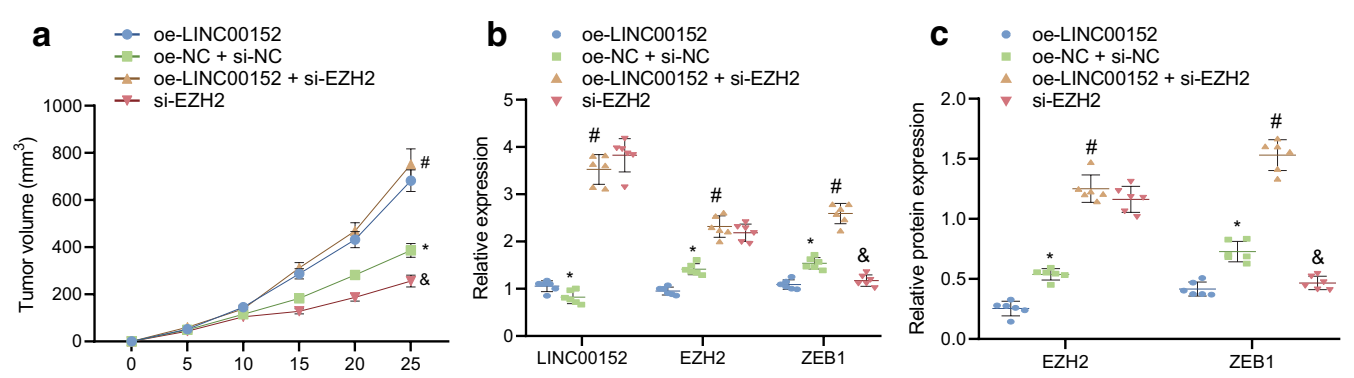

Fig. 6 LINC00152 mediates the EZH2/ZEB1 axis to promote EMT and EC cell resistance to L-OHP. a tumor growth rate curves of nude mice xenografted with EC cells $(n=6)$. $\mathbf{b}$ the mRNA expression of LINC00152, EZH2, and ZEB1 in tumor tissues of nude mice xenografted with EC cells $(n=6)$ detected by RT-qPCR. $\boldsymbol{c}$ the protein expression of EZH2 and ZEB1 in tumor tissues of nude mice xenografted with EC cells $(n=6)$ detected by Western blot analysis. ${ }^{*} p<0.05$ vs. The oe-NC + si-NC group; \# $p<0.05$ vs. the oe-NC + si-NC + L-OHP group; $\& p<0.05$ vs. the oe-LINC00152 + si-NC+ L-OHP group. Data are presented as mean \pm standard deviation of three technical replicates

EC cells to L-OHP, thus reducing chemoresistance. We found that administration of si-ZEB1, by inducing overexpression of LINC00152, could reverse the effects of LINC00152 on EMT and resistance of EC cells to L-OHP.

\section{Discussion}

Despite optimal current treatment, the survival rate of ESCC remains rather low due to its aggressive invasion and metastasis as well as the resistance to chemotherapy [1]. Emerging evidences have revealed that lncRNAs can be involved in tumorigenesis and development of EC [18, 32]. It has been reported that LINC00152 plays a crucial role in EMT and chemo-resistance in breast cancer cells [33]. Besides, LINC00152 was proven to contribute to resistance of colon cancer cell to L-OHP treatment [19]. Here, present study was conducted to explore the regulatory role of LINC00152 in L-OHP-induced EMT and resistance in $\mathrm{EC}$, as well as testing the involvement of ZEB1.

The first finding in this study was that LINC00152 was highly expressed in EC cells, which is consistent with earlier results in gastric cancer [34] and ESCC [35]. Subsequently, we designed gain- and loss-of-function experiments to explore its effects on biological process of EC cells. Results indicated that LINC00152 contributed to enhanced EMT and resistance to L-OHP of EC cells, which concurred with findings in previous studies. For example, one previous study demonstrated that LINC00152 confers chemo-resistance of colorectal cancer cells to 5-FU treatment [36], and that LINC00152 is conductive to chemosensitivity of colon cancer cell to L-OHP [19]. Additionally, LINC00152 contributes to EMT in glioblastoma [37]. These literature results collectively suggested that LINC00152 played a promotive role in EMT and drug resistance of cancer cells. In contrast, inhibition of LINC00152 suppresses EMT and resistance of chemotherapy, as verified in breast cancer [33].
However, details of the regulatory mechanisms needed further clarification. Subsequently, our present investigation revealed that LINC00152 was capable of facilitating EMT and resistance of EC cells to L-OHP via interacting with $\mathrm{EZH} 2$, which is partially corroborating previous results that LINC00152 facilitates EMT in colon cancer by interaction with $\beta$-catenin.

$\mathrm{EZH} 2$ is an enhancer of H3K27me3, alteration of which are considered to be carcinogenic drivers of lymphoma and other malignancies [38]. This work confirmed that EZH2 was highly expressed in EC cells, concurring with its known expression pattern in ESCC [39]. More importantly, loss of EZH2 reversed induction of EMT and resistance of EC cells to L-OHP treatment, manifesting in reductions in vimentin, and increase expression of E-cadherin, cleaved PARP and cleaved Caspase 3. These results were partially consistent with a previous finding that LINC00152 diminished expression of E-cadherin via interacting with EZH2, thereby promoting EMT in hepatocellular carcinoma [40]. Additionally, a few studies have found that many lncRNAs exert pro-tumorigenic effects via interaction with EZH2. For instance, IncRNA H19 contributes to progression of tongue squamous cell carcinoma through interaction with EZH2 [41]. Also, IncRNA MALAT1 promotes EMT of EC through EZH2 cell [42]. Based on previous and present results, we conclude that LINC00152 contributes to EMT and resistance of EC cells to L-OHP via interaction with EZH2.

Further, we saw high expression in EC cells of ZEB1, which is proved to be an oncogene in various cancers, including colorectal cancer [40] and lung cancer [43], as well as ESCC [44]. Besides, IncRNA CASC15 was found to promote EMT in gastric cancer by targeting ZEB1 [45]. More importantly, it has been confirmed that IncRNA MALAT1 can release EZH2 by binding to PRC2 complex, of which EZH2 is a main component thus blocking transcriptional inhibition of downstream genes 


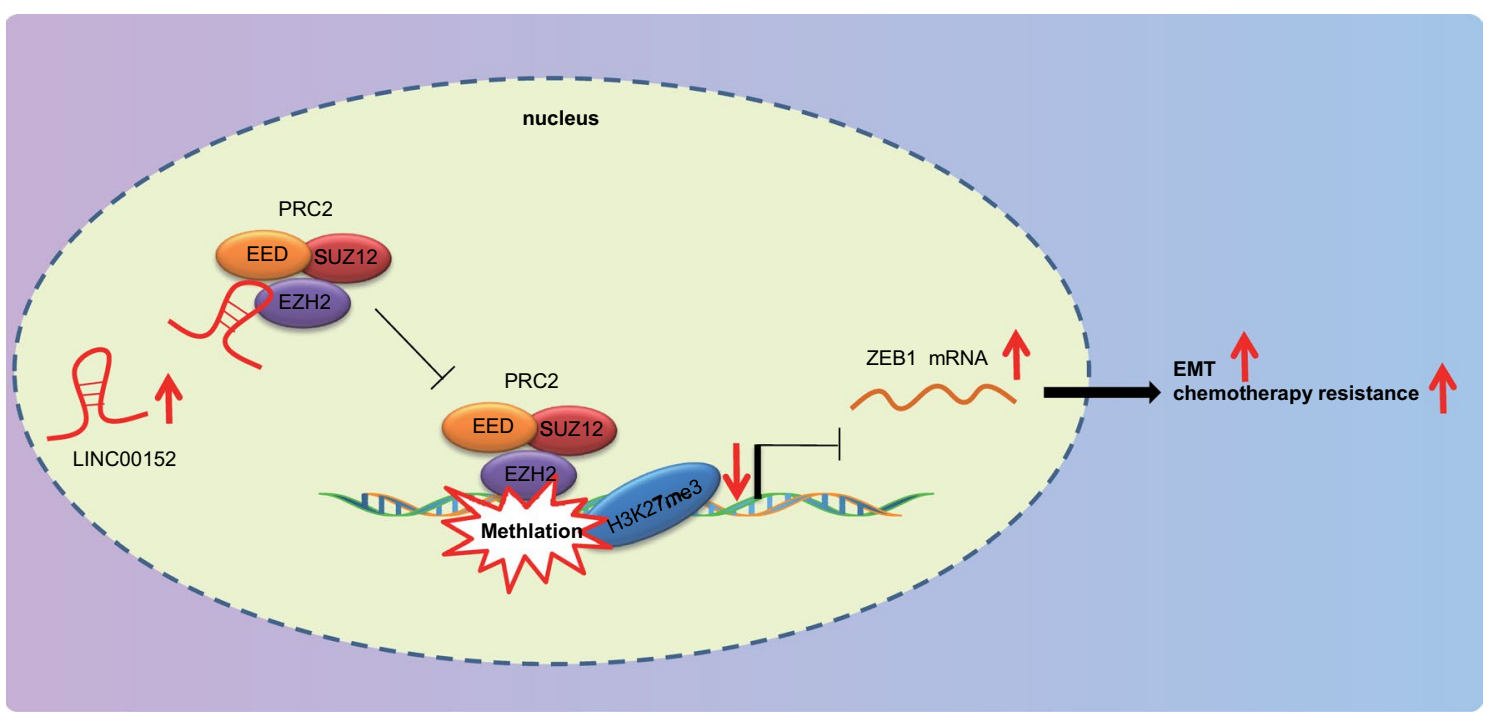

Fig. 7 The mechanism graph of regulatory network and function of LINC00152/EZH2/ZEB1. The highly expressed LINC00152 in EC could bind to the PRC2 protein complex (EZH2) to hinder the transcriptional inhibition of the downstream gene ZEB1 and increase the expression of ZEB1 through interaction with $\mathrm{EZH} 2$. The overexpression of ZEB1, on the one hand, increases the vimentin (interstitial protein marker) expression and decreases E-cadherin (epithelial protein marker) expression, thereby accelerating EMT; meanwhile, LINC00152 reduces the cleaved PARP and cleaved Caspase 3 expression, resulting in EC cell resistance to L-OHP

and consequently promoting the expression of HIV-1 in lung adenocarcinoma [28]. Additionally, lncRNA DANCR upregulates FBP1 to accelerate proliferation and migration in cholangiocarcinoma via interacting with EZH2 [46]. Similarly, LINC00152 regulates expression of downstream gene IL24 via interacting with EZH2 in lung adenocarcinoma [20]. In accord with those findings, our study suggests that LINC00152 enhanced EMT and resistance of EC cell to L-OHP by upregulating ZEB1 through interaction with EZH2.

\section{Conclusions}

To conclude, present findings collectively suggest that LINC00152 upregulated ZEB1 to enhance EC cell resistance to L-OHP and concomitant EMT through interaction with EZH2 (Fig. 7). Furthermore, silencing of LINC00152 led to impeded EMT and EC cell resistance to L-OHP. Thus, the present study contributes to identifying the role of LINC00152 in EC, offering a potential novel therapeutic target to repress EMT and resistance of EC cells to L-OHP.

\section{Limitation statement}

It is possible that cancer cell biology and signaling pathways will differ from that in tumors in vivo, which is the limitation of all the cell-line-based research. In this study, EC tissues were collected only for determining the expression of LINC00152. We did not document differences in the expression of LINC00152 in relation to response to L-OHP treatment in the patients.

\section{Abbreviations \\ EC: Esophageal cancer; EMT: Epithelial-mesenchymal transition; L-OHP: Oxali- platin; EZH2: Enhancer zeste homolog 2; EDTA: Ethylenediaminetetraacetic acid; OD: Optical density; RT-qPCR: Reverse transcription quantitative polymer- ase chain reaction; GAPDH: Glyceraldehyde-3-phosphate dehydrogenase; RIP: RNA immunoprecipitation; FISH: Fluorescence in situ hybridization.}

\section{Acknowledgements}

We would like show sincere appreciation to reviewers for critical comments on this article.

\section{Authors' contributions}

SZ, XH, SG, YW and XT designed the study. WL, WC, SL, CC and GQ collated the data, carried out data analyses. QW, XZ,WC and YJ produced the initial draft of the manuscript. ZP, ZH, YC and SC contributed to drafting the manuscript. All authors have read and approved the final submitted manuscript. All authors read and approved the final manuscript.

\section{Funding}

This study was supported by Third Batch and Fourth Batch of Medical and Health Science and Technology Program Project Funds in Shantou City in 2018 [Shan Fu Ke letter (2018) 238] and Dedicated Fund for Tumor-Targeting of Wu Jieping Medical Fundation supported (Project ID: 320.6750.19090-32).

\section{Availability of data and materials}

The datasets used and/or analysed during the current study are available from the corresponding author on reasonable request.

\section{Ethics approval and consent to participate}

The study protocol was approved by the Ethics Committee and Experimental Animal Ethics Committee of Cancer Hospital of Shantou University Medical College. All individuals signed informed written consent documents. Extensive efforts were made to ensure minimal suffering of the animals used in the study. 


\section{Consent for publication}

Not applicable.

\section{Competing interests}

The authors declare no competing interests.

\begin{abstract}
Author details
${ }^{1}$ Guangzhou Red Cross Hospital Affiliated of Ji-Nan University, Guangzhou 510220, P.R. China. ${ }^{2}$ Department of Pharmacology, Shantou University Medical College, Shantou 515000, P.R. China. ${ }^{3}$ Digestive Oncology, Cancer Hospital of Shantou University Medical College, Shantou 515000, P.R. China. ${ }^{4}$ Department of Pharmacy, Cancer Hospital of Shantou University Medical College, Shantou 515000, P.R. China.
\end{abstract}

Received: 9 October 2019 Accepted: 26 October 2020 Published online: 26 November 2020

\section{References}

1. Bray F, Ferlay J, Soerjomataram I, Siegel RL, Torre LA, Jemal A. Global cancer statistics 2018: GLOBOCAN estimates of incidence and mortality worldwide for 36 cancers in 185 countries. CA Cancer J Clin. 2018;68(6):394-424.

2. Zhang Y. Epidemiology of esophageal cancer. World J Gastroenterol 2013;19(34):5598-606.

3. Abnet CC, Arnold M, Wei WQ. Epidemiology of Esophageal Squamous Cell Carcinoma. Gastroenterology. 2018;154(2):360-73.

4. Guttmann DM, Mitra N, Bekelman J, Metz JM, Plastaras J, Feng W, et al. Improved Overall Survival with Aggressive Primary Tumor Radiotherapy for Patients with Metastatic Esophageal Cancer. J Thorac Oncol. 2017;12(7):1131-42.

5. Wang D, Plukker JTM, Coppes RP. Cancer stem cells with increased metastatic potential as a therapeutic target for esophageal cancer. Semin Cancer Biol. 2017:44:60-6.

6. Yoon DH, Jang G, Kim JH, Kim YH, Kim JY, Kim HR, et al. Randomized phase 2 trial of $\mathrm{S} 1$ and oxaliplatin-based chemoradiotherapy with or without induction chemotherapy for esophageal cancer. Int J Radiat Oncol Biol Phys. 2015;91(3):489-96.

7. Shen Z, Xu L, Li J, Zhang N. Capilliposide C. Sensitizes Esophageal Squamous Carcinoma Cells to Oxaliplatin by Inducing Apoptosis Through the PI3K/Akt/mTOR Pathway. Med Sci Monit. 2017;23:2096-103.

8. Shibue T, Weinberg RA. EMT, CSCs, and drug resistance: the mechanistic link and clinical implications. Nat Rev Clin Oncol. 2017;14(10):611-29.

9. Heery R, Finn SP, Cuffe S, Gray SG. Long Non-Coding RNAs: Key Regulators of Epithelial-Mesenchymal Transition, Tumour Drug Resistance and Cancer Stem Cells. Cancers (Basel). 2017:9(4).

10. Ge P, Cao L, Yao YJ, Jing RJ, Wang W, Li HJ. IncRNA FOXD2-AS1 confers cisplatin resistance of non-small-cell lung cancer via regulation of miR185-5p-SIX1 axis. Onco Targets Ther. 2019;12:6105-17.

11. Liu B, Wu S, Ma J, Yan S, Xiao Z, Wan L, et al. IncRNA GAS5 Reverses EMT and Tumor Stem Cell-Mediated Gemcitabine Resistance and Metastasis by Targeting miR-221/SOCS3 in Pancreatic Cancer. Mol Ther Nucleic Acids. 2018:13:472-82.

12. Silva A, Bullock M, Calin G. The Clinical Relevance of Long Non-Coding RNAs in Cancer. Cancers (Basel). 2015;7(4):2169-82

13. Kawasaki Y, Miyamoto M, Oda T, Matsumura K, Negishi L, Nakato R, et al. The novel InCRNA CALIC upregulates AXL to promote colon cancer metastasis. EMBO Rep. 2019;20(8):e47052.

14. Zhang CY, Li RK, Qi Y, Li XN, Yang Y, Liu DL, et al. Upregulation of long noncoding RNA SPRY4-IT1 promotes metastasis of esophageal squamous cell carcinoma via induction of epithelial-mesenchymal transition. Cell Biol Toxicol. 2016;32(5):391-401.

15. Kang M, Ren M, Li Y, Fu Y, Deng M, Li C. Exosome-mediated transfer of IncRNA PART1 induces gefitinib resistance in esophageal squamous cell carcinoma via functioning as a competing endogenous RNA. J Exp Clin Cancer Res. 2018:37(1):171.

16. Shi H, Shi J, Zhang Y, Guan C, Zhu J, Wang F, et al. Long non-coding RNA DANCR promotes cell proliferation, migration, invasion and resistance to apoptosis in esophageal cancer. J Thorac Dis. 2018;10(5):2573-82.
17. Chen JL, Lin ZX, Qin YS, She YQ, Chen Y, Chen C, et al. Overexpression of long noncoding RNA LINC01419 in esophageal squamous cell carcinoma and its relation to the sensitivity to 5 -fluorouracil by mediating GSTP1 methylation. Ther Adv Med Oncol. 2019;11:1758835919838958.

18. Hu HB, Jie HY, Zheng XX. Three Circulating LncRNA Predict Early Progress of Esophageal Squamous Cell Carcinoma. Cell Physiol Biochem. 2016;40(1-2):117-25.

19. Yue B, Cai D, Liu C, Fang C, Yan D. Linc00152 Functions as a Competing Endogenous RNA to Confer Oxaliplatin Resistance and Holds Prognostic Values in Colon Cancer. Mol Ther. 2016;24(12):2064-77.

20. Chen QN, Chen X, Chen ZY, Nie FQ, Wei CC, Ma HW, et al. Long intergenic non-coding RNA 00152 promotes lung adenocarcinoma proliferation via interacting with EZH2 and repressing IL24 expression. Mol Cancer. 2017;16(1):17.

21. Li W, Zheng J, Deng J, You Y, Wu H, Li N, et al. Increased levels of the long intergenic non-protein coding RNA POU3F3 promote DNA methylation in esophageal squamous cell carcinoma cells. Gastroenterology. 2014;146(7):1714-26 e5.

22. Comet I, Riising EM, Leblanc B, Helin K. Maintaining cell identity: PRC2-mediated regulation of transcription and cancer. Nat Rev Cancer. 2016;16(12):803-10

23. Wang Y, Li Y, He H, Wang F. Circular RNA circ-PRMT5 facilitates non-small cell lung cancer proliferation through upregulating $\mathrm{EZH} 2$ via sponging miR-377/382/498. Gene. 2019;720:144099.

24. Li H, Ma X, Yang D, Suo Z, Dai R, Liu C. PCAT-1 contributes to cisplatin resistance in gastric cancer through epigenetically silencing PTEN via recruiting EZH2. J Cell Biochem. 2019.

25. Wang FW, Ao X, Fu SM. The functional role of the EZH2 gene in controlling breast cancer stem cells. J BUON. 2019;24(3):1060-6.

26. D'Angelo V, lannotta A, Ramaglia M, Lombardi A, Zarone MR, Desiderio V, et al. EZH2 is increased in paediatric T-cell acute lymphoblastic leukemia and is a suitable molecular target in combination treatment approaches. J Exp Clin Cancer Res. 2015;34:83.

27. Ramaglia M, D’Angelo V, lannotta A, Di Pinto D, Pota E, Affinita MC, et al. High EZH2 expression is correlated to metastatic disease in pediatric soft tissue sarcomas. Cancer Cell Int. 2016:16:59.

28. Lehmann W, Mossmann D, Kleemann J, Mock K, Meisinger C, BrummerT, et al. ZEB1 turns into a transcriptional activator by interacting with YAP1 in aggressive cancer types. Nat Commun. 2016;7:10498.

29. Yang J, Tian B, Sun H, Garofalo RP, Brasier AR. Epigenetic silencing of IRF1 dysregulates type III interferon responses to respiratory virus infection in epithelial to mesenchymal transition. Nat Microbiol. 2017:2:17086.

30. Qu D, Sun WW, Li L, Ma L, Sun L, Jin X, et al. Long noncoding RNA MALAT1 releases epigenetic silencing of $\mathrm{HIV}-1$ replication by displacing the polycomb repressive complex 2 from binding to the LTR promoter. Nucleic Acids Res. 2019:47(6):3013-27.

31. Ma J, Zhang J, Weng YC, Wang JC. EZH2-Mediated microRNA-139-5p Regulates Epithelial-Mesenchymal Transition and Lymph Node Metastasis of Pancreatic Cancer. Mol Cells. 2018:41(9):868-80.

32. Aalijahan H, Ghorbian S. Clinical Application of Long Non-Coding RNAUCA1 as a Candidate Gene in Progression of Esophageal Cancer. Pathol Oncol Res. 2019.

33. Hu XL, Wang J, He W, Zhao P, Wu WQ. Down-regulation of IncRNA Linc00152 suppressed cell viability, invasion, migration, and epithelial to mesenchymal transition, and reversed chemo-resistance in breast cancer cells. Eur Rev Med Pharmacol Sci. 2018;22(10):3074-84

34. Pang Q, Ge J, Shao Y, Sun W, Song H, Xia T, et al. Increased expression of long intergenic non-coding RNA LINC00152 in gastric cancer and its clinical significance. Tumour Biol. 2014;35(6):5441-7.

35. Yang Y, Sun X, Chi C, Liu Y, Lin C, Xie D, et al. Upregulation of long noncoding RNA LINC00152 promotes proliferation and metastasis of esophageal squamous cell carcinoma. Cancer Manag Res. 2019;11:4643-54.

36. Bian Z, Zhang J, Li M, Feng Y, Yao S, Song M, et al. Long non-coding RNA LINC00152 promotes cell proliferation, metastasis, and confers 5-FU resistance in colorectal cancer by inhibiting miR-139-5p. Oncogenesis. 2017:6(11):395

37. Liu X, Yidayitula Y, Zhao H, Luo Y, Ma X, Xu M. LncRNA LINC00152 promoted glioblastoma progression through targeting the miR-107 expression. Environ Sci Pollut Res Int. 2018;25(18):17674-81.

38. Akpa CA, Kleo K, Lenze D, Oker E, Dimitrova L, Hummel M. DZNep-mediated apoptosis in B-cell lymphoma is independent of the lymphoma 
type, EZH2 mutation status and MYC, BCL2 or BCL6 translocations. PLoS One. 2019;14(8):e0220681.

39. He LR, Liu MZ, Li BK, Jia WH, Zhang Y, Liao YJ, et al. High expression of $\mathrm{EZH} 2$ is associated with tumor aggressiveness and poor prognosis in patients with esophageal squamous cell carcinoma treated with definitive chemoradiotherapy. Int J Cancer. 2010;127(1):138-47.

40. Zhang GJ, Zhou T, Tian HP, Liu ZL, Xia SS. High expression of ZEB1 correlates with liver metastasis and poor prognosis in colorectal cancer. Oncol Lett. 2013;5(2):564-8.

41. Zhang DM, Lin ZY, Yang ZH, Wang YY, Wan D, Zhong JL, et al. IncRNA H19 promotes tongue squamous cell carcinoma progression through betacatenin/GSK3beta/EMT signaling via association with EZH2. Am J Transl Res. 2017;9(7):3474-86.

42. Chen M, Xia Z, Chen C, Hu W, Yuan Y. LncRNA MALAT1 promotes epithelial-to-mesenchymal transition of esophageal cancer through Ezh2-Notch1 signaling pathway. Anticancer Drugs. 2018;29(8):767-73.

43. Zhang T, Guo L, Creighton CJ, Lu Q, Gibbons DL, Yi ES, et al. A genetic cell context-dependent role for ZEB1 in lung cancer. Nat Commun. 2016;7:12231.
44. Ma J, Zhan Y, Xu Z, Li Y, Luo A, Ding F, et al. ZEB1 induced miR-99b/let7e/miR-125a cluster promotes invasion and metastasis in esophageal squamous cell carcinoma. Cancer Lett. 2017;398:37-45.

45. Wu Q, Xiang S, Ma J, Hui P, Wang T, Meng W, et al. Long non-coding RNA CASC15 regulates gastric cancer cell proliferation, migration and epithelial mesenchymal transition by targeting CDKN1A and ZEB1. Mol Oncol. 2018;12(6):799-813.

46. Wang N, Zhang C, Wang W, Liu J, Yu Y, Li Y, et al. Long noncoding RNA DANCR regulates proliferation and migration by epigenetically silencing FBP1 in tumorigenesis of cholangiocarcinoma. Cell Death Dis. 2019;10(8):585

\section{Publisher's Note}

Springer Nature remains neutral with regard to jurisdictional claims in published maps and institutional affiliations.
Ready to submit your research? Choose BMC and benefit from:

- fast, convenient online submission

- thorough peer review by experienced researchers in your field

- rapid publication on acceptance

- support for research data, including large and complex data types

- gold Open Access which fosters wider collaboration and increased citations

- maximum visibility for your research: over $100 \mathrm{M}$ website views per year

At BMC, research is always in progress.

Learn more biomedcentral.com/submissions 